\title{
On-chip Low-threshold Silicon Nitride Distributed Feedback Colloidal Quantum Dot Laser
}

\author{
Yunpeng Zhu ${ }^{1,3}$, Weiqiang Xie ${ }^{1,3}$, Pieter Geiregat ${ }^{1,2,3}$, Suzanne Bisschop ${ }^{1,2,3}$, Tangi Aubert ${ }^{2,3}$, Edouard \\ Brainis $^{2,3}$, Zeger Hens ${ }^{2,3}$, Dries van Thourhout ${ }^{1,3}$ \\ ${ }^{1}$ Photonics Research Group, INTEC Department, Ghent University-IMEC, Ghent B-9000, Belgium \\ ${ }^{2}$ Physics and Chemistry of Nanostructures, Ghent University, Krijgslaan 281-S3, 9000 Ghent, Belgium \\ ${ }^{3}$ Center of Nano and Biophotonics (NB Photonics), Ghent University, Ghent B-9000, Belgium \\ Author e-mail address: yunpeng.zhu@ugent.be
}

\begin{abstract}
We report on hybrid integrated distributed feedback (DFB) lasers based on silicon nitride waveguide stacks containing a layer of embedded colloidal quantum dots. The DFB laser shows a low optical pumping threshold of $188 \mathrm{~kW} / \mathrm{cm}^{2}$ and operates in a single mode regime. Our results show the potential of colloidal QDs to hybridize silicon nitride photonic circuits and open the way to novel functionalities on said platform.

OCIS codes: (130.0130) Integrated Optics; (140.0140) Laser and Laser Optic
\end{abstract}

\section{Introduction}

Colloidal quantum dots (QDs) are nanometer sized pieces of inorganic, semiconductor crystal obtained through a wet chemical synthesis. Recent years saw a tremendous increase in their application potential due to improvements in surface chemistry, deposition methods, luminescence quantum yields and long term stability. Research in the past few years shows that QDs exhibit optical gain from visible to near infrared wavelengths, depending on the shape/size and composition of the QDs [1]. Thus, QDs are perfect candidates to realize the light emission and amplification functionalities for photonic integrated circuits (PICs).

Silicon nitride $(\mathrm{SiN})$ is considered to be a promising material to realize compact PICs due to its CMOS compatibility, relatively high refractive index ( 2.0) and broad transparency window. Numerous work has been done to demonstrate high quality passive functionality with SiN waveguide platform, but active components such as light sources are still largely absent. In previous work, we have already demonstrated that we can embed QDs into $\mathrm{SiN}$ layers without quenching their luminescence while maintaining a low-loss SiN waveguide platform [2]. Under femtosecond pulsed laser pumping, these embedded QDs also show amplified spontaneous emission (ASE) which can couple to the sandwich waveguide mode [3]. Recently, multimode disk lasers coupled to bus waveguides have been demonstrated within our hybrid QD SiN waveguide platform [4], which was the first demonstration of a fully integrated QD laser. However, the fabrication routine to achieve good waveguide coupling is quite extensive and the disk laser is inherently multimodal. We therefore aim at fabricating single mode devices.

In this work, we demonstrate on-chip and single mode operation of DFB type laser structures where the active gain medium is composed of colloidal quantum dots directly embedded into the body of the DFB. Instead of femtosecond pulses, the laser can be pushed to operation by nanosecond optical pumping, a great step forward for the hybrid QD-SiN PIC platform.

\section{Fabrication}
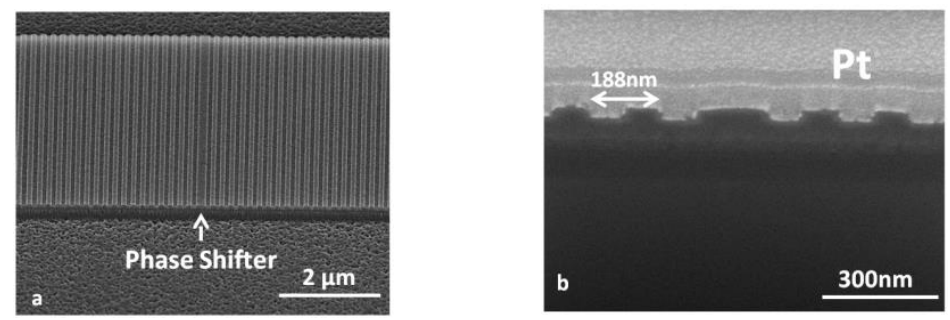

Fig.1. Colloidal Quantum Dot DFB laser (a) SEM picture of the fabricated QD DFB laser. (b)The cross section of the waveguide structure around the phase shifter region in the middle of the DFB laser, the periods and the phase shifter length is $188 \mathrm{~nm}$.

Starting from a silicon wafer with a $3 \mathrm{um}$ thermal oxide on top, a $75 \mathrm{~nm}$ thick SiN layer is deposited. The deposition is performed using an optimized plasma enhanced chemical vapor deposition (PECVD) process at a temperature of $270^{\circ} \mathrm{C}$. Next, "flash" CdSe/CdS core-shell QDs [5] are spin coated on top of this SiN layer to form a densely packed layer with a thickness of $c a .50 \mathrm{~nm}$. Another $90 \mathrm{~nm}$ thick SiN layer is deposited onto the QDs layer to form the full 
sandwich-type layer stack. Then, electron-beam (e-beam) lithography is used to define the DFB grating itself. A reactive ion etching (RIE) is performed to transfer the Ebeam patterning to the top SiN layer; the grating etching depth is about $35 \mathrm{~nm}$. Contact lithography followed by RIE are used to define the waveguides.

All the recipes have been optimized through our previous work where we obtained low-loss waveguides. The samples are cleaved before the waveguide RIE etching to ensure that the waveguides are terminated with a uniform facet. Fig.1 (a) shows a scanning electron microscope picture (SEM) of the fabricated device. Fig. 1 (b) shows the cross section of the waveguide structure around the phase shifter region in the middle of the DFB laser.

\section{Characterization and Results}

Characterization of the laser was done using a nanosecond pulsed laser system at $532 \mathrm{~nm}$ to excite the QD layer. The pumping laser is a Q-switched frequency doubled Nd:YAG laser with a 7 nanosecond pulse width at a $938 \mathrm{~Hz}$ repetition rate. The laser beam was expanded with a beam expander and then focused with a cylindrical lens onto the sample. The cylindrical lens can focus the pumping laser beam into a line shape of $\pm 2 \mathrm{~cm}$ by $80 \mu \mathrm{m}$. The beam is adjusted to overlap with the DFB laser and the light emitted from the DFB laser facet is collected by a multimode fiber and sent to a spectrometer for analysis.

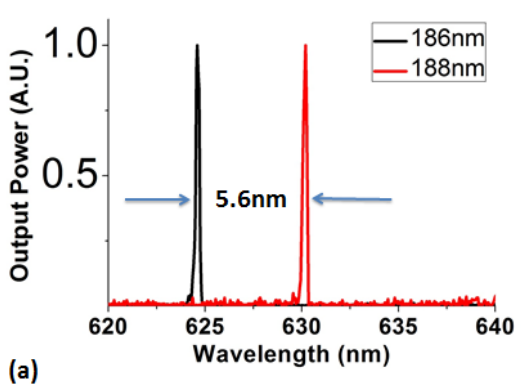

(a)

Fig. 2. (a) Emission spectrum with different DFB grating periods; (b) Log-scale Light(in)-Light(out) curve for $188 \mathrm{~nm}$ period DFB laser; the inset is a linear scale plot

Fig. 2. (a) shows the emission spectrum with different DFB grating periods. The two lasers with different grating periods are lasing single mode at $624.6 \mathrm{~nm}$ and $630.2 \mathrm{~nm}$ separately. The peak FWHM is around $0.2 \mathrm{~nm}$. In Fig. 2. (b) we plot the total output intensity versus the pump fluence for a DFB laser with a $188 \mathrm{~nm}$ period. From the measurement, we can estimate the laser threshold is around $1.4 \mathrm{mw}$, results in a threshold power density around 53.3 $\mathrm{kw} / \mathrm{cm}^{2}$. Moreover, the measurement result shows a well-defined S-Shaped curve indicative good laser operation.

Although previous reports show that lasing with QDs is possible [6], this result is the first demonstration of nanosecond pumped lasing of a fully integrated single-mode lasing structure. For this type of QDs, all non-radiative loss channels are faster than the laser pulse duration $7 \mathrm{~ns}$, so already this is a quasi-CW pumped QD laser.

\section{Conclusion}

We reported on the fabrication and characterization of a hybrid colloidal QD/SiN single mode DFB laser where the active QD gain medium is embedded into the DFB guiding layer. The laser showed single mode operation under nanosecond pumping with a low threshold density of ca. $50 \mathrm{~kW} / \mathrm{cm}^{2}$. Our results show the potential of colloidal QDs to hybridize silicon nitride photonic circuits and open the way to novel functionalities on said platform. Future steps are to realize quasi-continuous wave pumping through optimization of the cavity design and fabrication; and use of other types of inorganic nanomaterials as active gain medium such as CdSe platelets [7].

This work is partly supported by the EU through the projects ERC-ULPPIC and ICT-NAVOLCHI and by the Belgian Science Policy Office (IAP). P.G. acknowledges the FWO-Vlaanderen for support.

\section{References}

[1] Klimov, Victor I., et al. "Single-exciton optical gain in semiconductor nanocrystals." Nature 447.7143 (2007): 441-446.

[2] Xie, Weiqiang, et al. "Low-loss silicon nitride waveguide hybridly integrated with colloidal quantum dots." Optics Express 23.9 (2015):

$12152-12160$.

[3] Zhu, Yunpeng, et al. "Hybrid Colloidal Quantum Dot Silicon Nitride Waveguide Gain Measurement Based on Variable Stripe Length

Method." CLEO: Applications and Technology. Optical Society of America, 2016.

[4] Xie, Weiqiang, et al. "Integrated Silicon Nitride Microdisk Lasers Based on Quantum Dots." CLEO: Science and Innovations. Optical Society of America, 2016..

[5] Cirillo, Marco, et al. "“Flash" Synthesis of CdSe/CdS Core-Shell Quantum Dots." Chemistry of Materials 26.2 (2014): 1154-1160.

[6] Adachi, Michael M., et al. "Microsecond-sustained lasing from colloidal quantum dot solids." Nature communications 6 (2015).

[7] She, Chunxing, et al. "Low-threshold stimulated emission using colloidal quantum wells." Nano letters 14.5 (2014): $2772-2777$. 